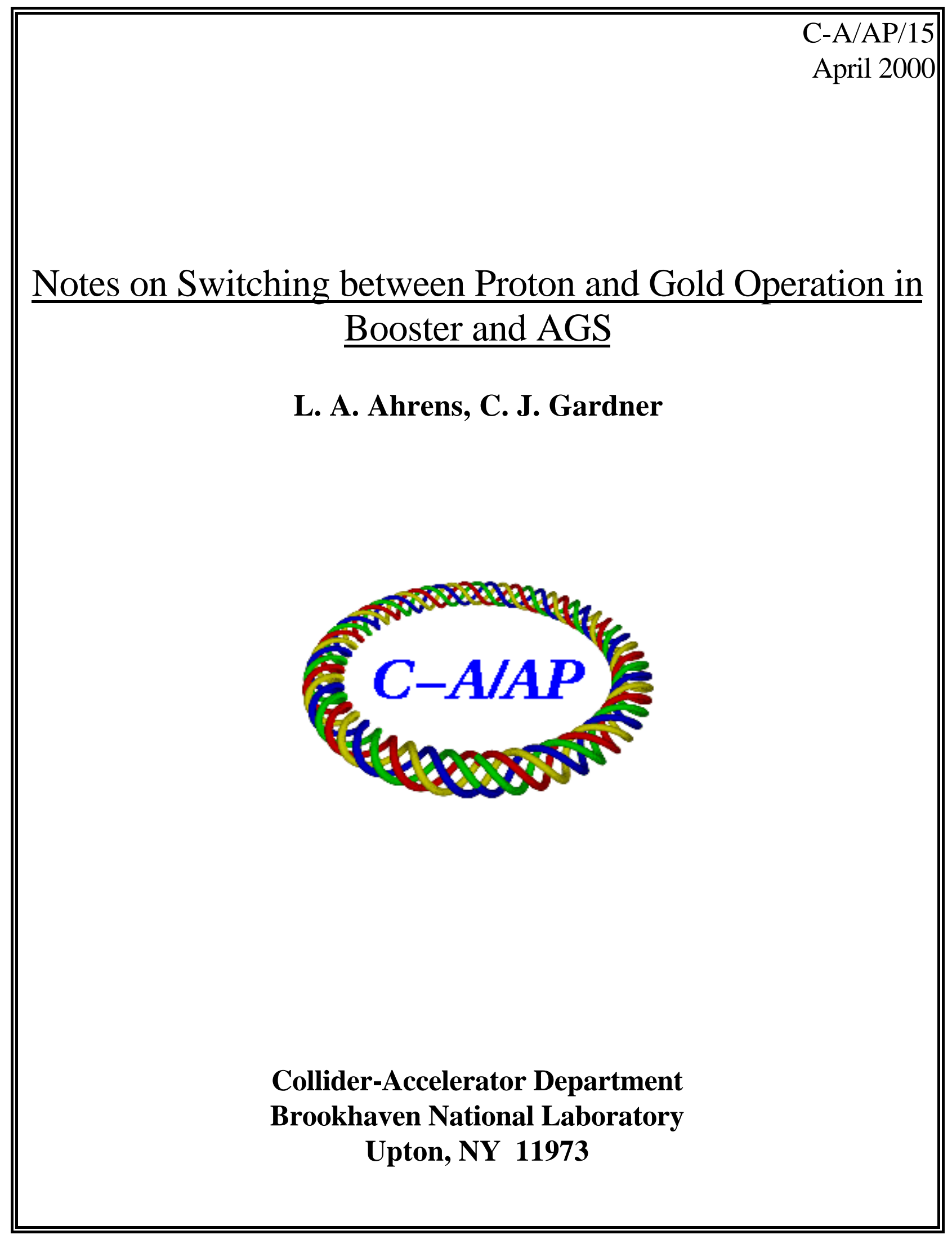




\title{
Notes on Switching between Proton and Gold Operation in Booster and AGS
}

\author{
L.A. Ahrens and C.J. Gardner
}

April 13, 2000

In order to utilize the high-intensity proton capabilities of the Booster and AGS during RHIC operation, we need to be able to switch back and forth between proton and gold operation quickly and efficiently. Switching times of 15 minutes or less are desired. Early this year, three days were taken out of the HEP program to switch between high-intensity proton and gold operation and to identify all items that require different settings or configurations for the two modes of operation. In particular, items which currently can not be switched in the desired time were identified. Following are some notes on the switching exercise and the items identified.

\section{Synopsis of Switching Exercise}

\section{$1.1 \quad$ Monday 31 January 2000}

- 04:00. HEP (Muon g-2) OFF. After Cooldown, Booster ring accessed for maintenance. Radiation damaged Optical-Coupler for profile monitor 29MW141 replaced. PUE C4 Checkout.

- Booster RF modifications completed in 2 hours.

- Maintenance items (Booster vacuum leak repair, etc.) consumed most of the daytime hours.

- 17:00. TTB Beamstops opened.

- 17:45. Beam transport tuned to inflector.

- 18:00. Two turns of beam "notch" seen on PUE D1. 
- 22:30. After tuning for 2.5 hours, Zeno has $3 \mathrm{~ms}$ survival of spiraling beam. Measure revolution frequency.

\subsection{Tuesday 1 February 2000}

- 02:00. Zeno finished tuning for the night. With $400 \mu \mathrm{s}, 25 \mu \mathrm{A}$ pulse from Tandem, he has $1.5 \times 10^{9} \mathrm{Au}^{32+}$ ions stacked in Booster with $0.75 \times 10^{9}$ surviving $1 \mathrm{~ms}$ later.

- 04:30. Acceleration to Extraction, and Extraction Setup.

- 09:00. Extraction Setup.

- Forgot to put C6 foil holder in "Blank" position! This may have hampered Booster injection setup.

- Problems trying to inject into AGS. This was later found to be due to a mis-communication regarding the setup of the L20 inflector for heavy-ion running. (When we ran Gold for RHIC in 1999, L20 was put in the "Proton" mode. For this run it was put in the "Heavy-Ion" mode and therefore required a different setting.)

- 11:30. Go to 4 Tandem pulses per AGS cycle. AGS injection frequency measured.

- 23:30. Four transfers injected onto AGS Porch.

\subsection{Wednesday 2 February 2000}

- 09:00. Beam accelerated to full energy in AGS during the wee hours. AGS is able to capture all 6 bunches per Booster cycle!

- 12:30. Ahrens, et. al. setup correct AGS extraction field and measure RF frequency there.

- 14:00. Beam seen on H10 Flag.

- 15:45. H10 Septum will not turn ON! Decide to "pack it in" and begin switch back to proton running. 


\section{Proton-Gold Switching Items}

\section{$2.1 \quad$ Booster Items}

1. TTB Profile Monitor Electronics. The electronics modules for TTB profile monitor 29MW141 are located in a yellow cabinet in the Booster ring near the C3 inflector. One component in the modules, an optical coupler used to insert and retract the profile monitor, is particularly susceptible to radiation damage during high-intensity proton runs. Switching from Proton to Gold operation usually requires a ring entry to replace this component. Other components in the cabinet seem to be less susceptible to radiation. Tony Curcio suggests that the cabinet be moved to a location outside the Booster ring to allow for easy access and maintenance. This of course means longer cables between the profile monitor and electronics which may degrade the signal quality. Another option might be to better shield the electronics in the present location.

2. Inflector Position. In the past we have made ring accesses to move the downstream end of the C3 inflector inward to the "inserted" position for heavy-ion operation and outward to the "retracted" position for high-intensity proton operation. The difference between the two positions is 0.25 inches at the downstream end of the inflector. During the FY2000 Muon g-2 run we found that there is no problem leaving the $\mathrm{C} 3$ inflector "inserted" during high-intensity proton operation provided that losses there are monitored closely and appropriate local orbit bumps are put in place to keep the losses as low as possible. With these provisions then, the inflector may be left in the position normally used for heavy-ion operation thereby eliminating the need for ring accesses.

3. Inflector Insulation. The inflector has an elbow-shaped high-voltage feed-through in the Booster ring which is normally filled with an insulating fluid during heavy-ion operation. Experience has shown that the fluid is degraded by radiation during proton operation; it loses its insulating properties and becomes sticky, making it difficult to clean from inside wall of the feed-through. The fluid is therefore normally drained prior to high-intensity proton operation. Tom Russo has found that the inflector voltage can be brought up to $30 \mathrm{KV}$ with no fluid in the elbow. This is sufficient for 
Gold operation which requires just $23 \mathrm{KV}$. However, Iron ions for NASA's Biology program require $50 \mathrm{KV}$. If, in the future, we wish to switch between Proton and Iron operation, the inflector will require some kind of feed-through that is not damaged by radiation.

4. "Special" BPMs. For both Proton and Heavy-Ion operation, special amplifiers are connected to a small number of BPMs in the Booster ring. These are used to measure the betatron tunes and to obtain position-versus-turn data during injection. Typically the BPMs at C4, D1, and B6 have been used for the position information while those at E5 and E8 are used for the tunes. The associated electronics, which must reside near the BPMs, are susceptible to radiation damage during high-intensity proton operation. The component particularly susceptible is the device that switches between various amplifier gains; the amplifiers themselves seem to be more robust. Switching from Proton to Heavy-Ion operation usually requires a ring entry to replace the switching component. One option here is to leave the amplifiers on the above BPMs in the high-gain mode required for heavy-ion operation, and use other BPMs for Proton measurements.

5. Booster-Input Monitor. This is a scaler that registers the integrated beam current from current transformer NX58 for proton injection and from 29XF104 for heavy-ion injection. (NX58 is located in the Linac HEBT line; 29XF104 is located near the end of the TTB line.) When switching from proton to heavy-ion operation, the scaler input has to be switched from NX58 to 29XF104. This requires several steps and adjustments as detailed by Gary Smith [1].

6. Circulating-Beam Monitor. This is a set of scalers that register the amount of beam circulating in the Booster at various times during the magnetic cycle. The input for the scalers is the C6 current transformer. Switching between proton and heavy-ion operation requires several steps as detailed by Gary Smith [1].

7. Injection Current Transformer. This is part of the C6 current transformer package. Its gain must be switched on the relavant spreadsheet page when switching between proton and heavy-ion operation. This is a PPM operation, but the signal from high-intensity protons on one Booster cycle can saturate the high-gain amplifier used to observe heavy-ions on a subsequent cycle. 
This is not a problem if the time between the proton and heavy-ion cycles is more than a few seconds.

8. C6 Injection Foil Holder. When switching from proton to heavy-ion operation, the $\mathrm{C} 6$ foil holder needs to be rotated to the "blank" position. This is initiated by a spreadsheet command; the mechanical action then requires several AGS cycles to complete. The spreadsheet operation could become part of a computer application for switching between proton and heavy-ion operation.

9. Booster Injection Bumps. These can be switched between proton and heavy-ion configurations on a PPM basis. This was demonstrated in January 1999.

10. Booster RF System. Significant modifications, as detailed by Tom Hayes [2], are required to switch between proton and heavy-ion acceleration. Ring entry is not required.

11. Glitch Bit. K. Zeno has shown that the timing of the tune quadrupoles is critical during injection of heavy ions in Booster. To eliminate jitter in the timing, a feature called a "glitch bit" (also called an "Interruptable Vector") was recently added to the tune control program. This, however, (for reasons not entirely understood) causes the dwell field on the first few Booster cycles to be different from the field on subsequent cycles. Zeno has circumvented the problem by using real-time rather than gauss clock triggers for heavy-ion injection. This works well, and switching between the real-time and gauss clock modes is a spreadsheet operation. The only complication is that some RF events also need to be switched to real-time triggers and this currently requires a wiring change in Building 914. For the Beam-Control-Start and Radial-Loop-Start events, Kevin Smith has implemented a solution where real-time and gauss clock triggers are OR'd together to produce the desired event. Switching between real-time and gauss clock for these events then becomes a spreadsheet operation.

12. F3 Kicker and F6 Septum. These require different settings (a spreadsheet operation) but do not require any special changes when switching between proton and heavy-ion operation. 


\subsection{BTA Items}

1. BTA Multiwire Profile Monitors. These need different gain settings for proton and heavy-ion operation. These can be set on the relavant spreadsheet page or in the Beamline Instrument Application.

2. BTA Magnets. These need different spreadsheet settings for transport of protons and heavy ions; subsequent tuning to optimize transport is usually required. They do not operate on a PPM basis. T. Roser suggests that we may need to cycle the magnets in order to get reproducible fields for given setpoints. This process, if it can be specified, could then be automated. (This could become part of a computer application for switching between proton and heavy-ion operation.)

3. BTA Stripping Foil. When switching from proton to heavy-ion operation, the foil holder in the BTA line needs to be rotated from the "blank" position (used for protons) to the position with the appropriate stripping foil for heavy-ions. This is initiated by a spreadsheet command; the mechanical action then requires several AGS cycles to complete. The spreadsheet operation could become part of a computer application for switching between proton and heavy-ion operation.

\subsection{AGS Items}

1. L20 Inflector. In the past, the L20 inflector has been set up in "Proton" and "Heavy-Ion" modes for proton and heavy-ion operations respectively. The heavy-ion mode essentially allows the inflector current to be set with sufficient resolution at the lower values required for heavy-ion operation. Switching between modes involves connecting or disconnecting (by hand) an auxiliary inductor in the L18 house. (The inductor is connected in series with the inflector for the lower currents.) During the last Gold run (Summer 1999) the inflector was left in the proton mode and it was found that the current still could be adjusted and set with sufficient resolution for gold operation. Setting the current is a spreadsheet operation. For currents lower than those required for gold, connecting the inductor in series with the inflector may be necessary.

2. A5 Kicker. The setup of the PFN for the A5 kicker is different for 
Proton and Gold operation. Proton operation requires a "Tail Biter" while Gold operation requires that this feature be turned OFF.

Turning the "Tail Biter" back ON requires a 15 min warm-up period for the thyratron tube switch that initiates the tail bite.

3. A20 Profile Monitor. The A20 multiwire profile monitor is invaluable for setting up spiraling gold beam in AGS. The drive mechanism must be enabled for heavy-ion operation and appropriately disabled (to protect the wires from instant destruction) during high-intensity proton operation. J.W. Glenn suggests that the drive could be disabled automatically when the LTB beamstops are opened. (Enabling the drive for low-intensity proton or polarized proton operation would then require that this feature be overridden.)

4. G5 Wall Monitor. Switching from Proton to Gold operation requires that a $20 \mathrm{~dB}$ attenuator be removed. This is presently done by hand in the MCR. W. van Asselt suggests that a computer controlled coaxial switch would be useful for this operation.

5. AGS Injection Current Transformer. This transformer has no active computer control. The gain is set by a front panel switch on the board in the A10 House.

6. AGS Circulating-Beam Monitor. Switching from proton to heavy-ion operation requires switching from the CERN transformer at F15 to the Bergoz transformer at A20. (The Bergoz was supposed to replace the CERN transformer, but was found to have problems operating above $40 \mathrm{TP}$ during proton operation.) The switch from CERN to Bergoz requires several steps as detailed by Gary Smith [1].

7. AGS Orbit Acquisition System. In the past, $20 \mathrm{~dB}$ attenuators in three of the AGS Ring Houses have been connected and removed (by hand) for high-intensity proton and heavy-ion operation respectively. Kevin Smith believes that $12 \mathrm{~dB}$ attenuators will work for both proton and heavy-ion operation.

8. "Special" BPM's. In the past, prior to heavy-ion operation, a ring entry has been made to connect special high-gain amplifiers to a few of the BPMs. These are needed for the AGS tune meter and to provide position-versus-turn information on the injection porch. Although these electronics (presumably) can not survive in the high-intensity proton environment, the amplifiers for the damper 
BPMs at F20 do survive and can provide the desired position-versus-turn signals. Could similar electronics be employed on dedicated BPMs for the tune meter??

9. AGS Ring Loss Monitor System. Switching the gains is a spreadsheet operation.

10. AGS RF system. Significant modifications, as detailed by Tom Hayes [2], are required to go from proton to heavy-ion acceleration. Ring entry is not required.

11. AGS Transverse Damper. This would be useful for damping the transverse osillations of heavy-ion bunches injected off the equilibrium orbit. Presently this device does not work at the RF harmonic required for heavy-ion capture.

12. AGS Extraction Kicker and Septum. Different spreadsheet settings are required for proton and heavy-ion operation, but no special modifications are necessary.

\section{$2.4 \quad$ ATR Items}

1. U Line Magnets. Setting these magnets is a spreadsheet or pet page operation. Do we need to cycle the magnets in order to get reproducible fields for given setpoints??

2. U Line Current Transformer. The only transformer in U line used for both proton (muon g-2) and heavy-ion operation is UXF1. This is located just downstream of the H10 septum. (So close, in fact, that beam gets through it even if the setting of the septum is only roughly optimized.) For proton operation, the amplifier gain is set to " $6 \mathrm{~dB}$ " on the relavant pet page and $12 \mathrm{~dB}$ of attenuation is added (connected by hand in the A trailer) to the amplifier input. For heavy-ion operation, the $12 \mathrm{~dB}$ of attenuation is removed and the pet page gain is set to " $24 \mathrm{~dB}$ ". (One can leave the gain setup in the proton mode with some degradation to the gold measurement.)

3. U Line BPM's. The first four BPMs in the U Line are used for both proton (muon g-2) and heavy-ion operation. Switching the gains is a pet page operation. 
4. U Line Flags and TV Cameras. At least one camera has to be changed for heavy-ion opertation. Could we live without it for gold operation??

5. U Line Loss Monitors. The U-Line loss monitors are used for both proton (muon g-2) and heavy-ion operation. Switching the gains is a pet page operation.

\subsection{Radiation Security and Other Items}

1. Appropriate measures must be taken to ensure that high-intensity proton beam can not go where only heavy-ions are allowed! According to Neville Williams, the 8-degree and 20-degree bends in the ATR Line are turned OFF by the Radiation Security System when the LTB Beamstops are opened.

2. The appropriate default user must be set for proton and for heavy-ion operation.

3. Timed archives must be invoked for proton and heavy-ion operation.

4. Several of the items listed in these notes involve spreadsheet or pet page operations. These could all become part of a computer application for switching between proton and heavy-ion operation.

\section{Summary of Items that Can Not at Present be Switched Quickly and Efficiently}

1. TTB Profile Monitor Electronics. Booster Ring entry required to replace radiation damaged component.

2. Booster-Input Monitor. Switching requires several steps.

3. Booster Circulating-Beam Monitor. Switching requires several steps.

4. Booster RF System. Significant modifications required.

5. "Special" Booster BPMs. Booster Ring entry required to deploy high-gain amplifiers. 
6. AGS A5 Kicker. 15 minute warm-up period when switching back to proton operation.

7. AGS Injection Current Transformer. This transformer has no active computer control.

8. AGS Circulating-Beam Monitor. Switching requires several steps.

9. AGS RF system. Significant modifications required.

10. "Special" AGS BPM's. AGS Ring entry required to deploy high-gain amplifiers.

11. U Line Current Transformer. Attenuator connected by hand in the A trailer.

12. U Line TV Camera. Camera needs to be deployed for heavy-ion operation.

\section{References}

[1] G.A. Smith, "Quick Mode Change for Beam Intensity Monitors", February 17, 2000.

[2] T.P. Hayes, J.M. Brennan, and K.S. Smith, forthcomimg note. 